\title{
IGF-1 Can Either Protect Against or Increase LPS-Induced Damage in the Developing Rat Brain
}

\author{
YI PANG, BAOYING ZHENG, LEIGH R. CAMPBELL, LIR-WAN FAN, ZHENGWEI CAI, AND PHILIP G. RHODES
}

Department of Pediatrics, University of Mississippi Medical Center, Jackson, Mississippi 39216

\begin{abstract}
Periventricular leukomalacia (PVL) is a major form of brain damage in premature infants. This study was to test whether IGF-1 can prevent PVL-like brain damage induced by lipopolysaccharide (LPS) in the neonatal rat. Intraventricular delivery of LPS resulted in an acute brain inflammatory response, i.e., rapid recruitment of polymorphonuclear leukocytes (PMNs), activation of microglia and astrocytes, and induction of IL- $1 \beta$ (IL1 $\beta$ ) expression. Brain inflammation was associated with the loss of $\mathrm{O} 4+$ preoligodendrocytes (preOLs), a decrease of myelin basic protein (MBP) in the white matter and an increase of pyknotic cells in the cortex. IGF-1 at a low dose significantly prevented LPS-induced deleterious effects without alteration of IL-1 $\beta$ expression and microglia/astrocytes activation. On the other hand, the low dose of IGF-1 enhanced LPS-induced PMNs recruitment and blood-brain barrier $(\mathrm{BBB})$ permeability, and caused intracerebral hemorrhage. At higher doses, co-application of IGF-1 with LPS resulted in a high mortality rate. Brains from the surviving rats showed massive PMN infiltration and intracerebral hemorrhage. However, these adverse effects were not found in rats treated with IGF-1 alone. This study provides the alarming evidence that in an acute inflammatory condition, IGF-1 may have severe, harmful effects on the developing brain. (Pediatr Res 67: 579-584, 2010)
\end{abstract}

$\mathrm{P}_{\mathrm{p}}^{\mathrm{e}}$ eriventricular leukomalacia (PVL) is the major neuropathologic form of brain injury in preterm infants and is the leading cause of cerebral palsy (1). The pathogenesis of PVL is not completely understood, but hypoxia-ischemia and infection/inflammation have been suggested as two primary factors $(2,3)$. Several animal models have been developed based on either hypoxia-ischemia or inflammation. We previously reported that intracerebral delivery of lipopolysaccharide (LPS) preferentially induces white matter damage, loss of immunoreactivity of immature OL markers, increased size of lateral ventricles, and hypomyelination (4).

Preoligodendrocytes (preOLs) are preferentially damaged in PVL (1,5); therefore, strategies in protecting preOLs against hypoxic-ischemic or inflammatory damage are critical in developing therapies to treat PVL. IGF-1 seems to be a plausible candidate for such a purpose due to its potent survival effect. In vitro studies have shown that IGF-1 is able

Received October 8, 2009; accepted February 26, 2010.

Correspondence: Philip G. Rhodes, M.D., Department of Pediatrics, Division of Newborn Medicine, University of Mississippi Medical Center, 2500 N. State Street, Jackson, MS 39216; e-mail: prhodes@ped.umsmed.edu

Supported in part by HD 35496 from the National Institute of Child Health and Human Development; by NS 54278 from the National Institute of Neurological Disorders and Stroke; and by funds from the Newborn Medicine Division and the Department of Pediatrics, University of Mississippi Medical Center, Jackson, Mississippi. to rescue OLs from various insults, including TNF $\alpha$ cytotoxicity (6), growth factor deprivation (7), and excitotoxicity (8). In animal studies, IGF-1 has been shown to protect the developing brain against hypoxic-ischemic damage (9-11). However, it is not clear if IGF-1 can also protect the developing brain against inflammatory insults. Because of its potent survival and differentiating activity, IGF-1 is currently being tested as a therapy for a variety of nervous system pathologies. Therefore, the aim of this study was to test if IGF-1 can protect against LPS-induced brain white matter damage.

\section{MATERIALS AND METHODS}

Chemicals. LPS (055:B5) and fluorescence (FITC)-labeled albumin were obtained from Sigma Chemical Co. (St. Louise, MO). Recombinant human IGF-1 was from Cell Sciences (Canton, MA). Antibodies were obtained from the following sources: O4, myelin basic protein (MBP), glial fibrillary acidic protein (GFAP), and ED1 from Millipore (Temecula, CA); CD43 from AbD Serotec (Raleigh, NC); APC-CC1 from Calbiochem (Gibbstown, NJ), and activated caspase- 3 from Cell Signaling Technology (Danvers, MA). The IL- $1 \beta$ (IL-1 $\beta$ ) ELISA kit was obtained from R\&D systems (Minneapolis, MN). The terminal deoxynucleotidyl TUNEL kit was obtained from Millipore.

Animal surgery and treatment. Although sex differences have become increasingly recognized to play a role in many diseases including cerebral palsy (12), we did not notice a significant sex difference in the LPS model. To be consistent with our previous studies, both sexes of Sprague-Dawley rat pups were used in the current study. LPS or IGF-1 was delivered intraventricularly to rat brain at postnatal day 5 (P5), as described elsewhere (13). Briefly, pups were anesthetized with isoflurane (4\% induction, $1.5 \%$ maintenance) and placed in a stereotaxic apparatus with an adapter for neonatal rats (David Kopf, Tujunga, CA). The intraventricular injection was performed using a $10 \mu \mathrm{L}$ syringe using the following coordination: $1.0 \mathrm{~mm}$ posterior and $1.0 \mathrm{~mm}$ lateral to the bregma, and $3.0 \mathrm{~mm}$ deep to the skull surface. LPS (10 $\mu \mathrm{g} / \mathrm{animal})$, IGF-1 $(0.5,5$, and $25 \mu \mathrm{g} / \mathrm{amimal})$, or IGF-1 in combination with LPS at a volume of $2 \mu \mathrm{L}$ was injected into the left lateral ventricle over a period of $5 \mathrm{~min}$. The control rats were injected with the same volume of sterile saline. The intraventricular delivery was confirmed in series coronary frozen sections from the rat brains that were injected with trypan blue. For histologic and immunohistochemistry studies, six animals were included in each treatment group.

At P6, P8, and P21, rat pup brains were fixed by transcardiac perfusion with $4 \%$ paraformaldehyde. Consecutive coronal brain sections were prepared for histology and immunohistochemistry studies. The experimental procedure was approved by the Institutional Animal Care and Use Committee at the University of Mississippi Medical Center and, in addition, was in accordance with the guidelines of the National Institutes of Health on the care and use of laboratory animals. Every effort was made to minimize the number of animals and their suffering.

Abbreviations: BBB, blood-brain barrier; GFAP, glial fibrillary acidic protein; H\&E, hematoxylin and eosin; IGF-1, insulin-like growth factor-1; LPS, lipopolysaccharide; MBP, myelin basic protein; PMN, polymorphonuclear leukocyte; Pre-OLs, pre-oligodendrocyte; PVL, periventricular leukomalacia; TUNEL, terminal deoxynucleotidyl transferase-mediated dUTP nick end labeling 
Histologic examination. Frozen sections $(10 \mu \mathrm{m})$ or free-floating sections (40 $\mu \mathrm{m}$, for $\mathrm{O} 4$ immunohistochemistry) were prepared at the level of bregma and dorsal hippocampus. At each level, 50 frozen sections and 20 free-floating sections in consecutive order were obtained. Hematoxylin and eosin (H\&E) staining were used to examine gross histopathology and hemorrhage, and Nissl staining was used to examine cell damage.

Immunohistochemistry and TUNEL. Immunohistochemistry was performed using standard protocols. After blocking, sections were incubated with primary antibodies for $2 \mathrm{~h}$ at room temperature (RT), the final dilution of the antibodies was as follows: GFAP (1:300), ED1 (1:200), CD43 (1:200), O4 (1:200), MBP (1:200), APC-CC1 (1:20), and active caspase-3 (1:100). After washing, sections were incubated with biotinylated second antibodies for $1 \mathrm{~h}$ at RT and then with FITC or Cy3-conjugated avidin for an additional $1 \mathrm{~h}$ in the dark at RT. Sections were then washed, dried, and mounted. Images were captured with a CCD camera (Oly-750, Olympus) and superimposed using the Adobe Photoshop (version 7.0) software, if necessary. For detection of cell death in the cortex at P6, sections were processed for TUNEL staining following the manufacturer's instruction.

FITC-labeled albumin leakage. Twenty-four $\mathrm{hr}$ after brain injection, the integrity of blood-brain barrier (BBB) was assessed using FITC-labeled albumin as described by Carvey et al. (14). Briefly, $0.5 \mathrm{~mL}$ of FITC-labeled albumin was infused into the left cardiac ventricle with the right atrium cut open. The descending circulation was clamped off during infusion. Within 2 min, the brains were removed and immediately immersed into Zambonie's fixative (7.5\% saturated picric acid, $12 \mathrm{mM} \mathrm{NaH}_{2} \mathrm{P}_{4}, 88 \mathrm{mM} \mathrm{Na}_{2} \mathrm{HPO} 4$ and $4 \%$ paraformaldehyde). Twenty-four hr later, the fixative was replaced with $30 \%$ sucrose protection solution for additional $2 \mathrm{~d}$. The brains were then serially cut into $30 \mu \mathrm{m}$ free-floating sections and immediately mounted onto slides for examination. Four animals were included in each treatment group.

ELISA to detect $I \boldsymbol{L}-1 \boldsymbol{\beta}$. The content of $\mathrm{IL}-1 \beta$ in the forebrain was measured by ELISA at $24 \mathrm{~h}$ after brain injection, when IL-1 $\beta$ was at its peak as shown by our previous study (4).

Quantification of histology and immunohistochemistry data. Three slides at the bregma level were selected for cell counting in each animal. O4+ and $\mathrm{APC}+$ cells were counted in the white matter where the positively labeled cells were most abundant. The mean value was calculated from three slides of the same animal. The number of CD43 and ED1 positive cells was counted in the entire white matter track and averaged. MBP immunohistochemistry was quantified using a grading method, and the score was from 0 to 3 , where 0 , no staining; 1 , weak but detectable staining; 2 , reduced staining compared with 3 but stronger than 1 ; and 3, normal

Statistics. Unless otherwise indicated, results are represented as mean \pm SEM. One Way Analysis of Variation followed by Student-Newman-Keuls test was used to determine statistical significance among treatments, and the level of significance was set at $p<0.05$.

\section{RESULTS}

IGF-1 at a low dose prevented LPS-induced brain injury. Intracerebral injection of LPS resulted in a loss of preOLs, reduced MBP immunostaining in the white matter, and increased number of pyknotic cells in the cortex. The number of $\mathrm{O} 4+$ preOLs in the white matter was significantly reduced by LPS (Fig. $1 B$ and $E$ ) compared with that in the control (Fig. $1 A$ and $D$ ) at $\mathrm{P} 8$. To address the question whether the loss of $\mathrm{O} 4$ immunoreactivity is due to degeneration of preOLs, sections were double labeled with $\mathrm{O} 4$ and active caspase- 3 antibodies. As shown in Fig. 1G-I (arrow heads), some O4+ cells colocalize with active caspase-3, indicating that they were under degeneration (the colabeled cells account for $15.6 \pm 2.3 \%$ of total $\mathrm{O} 4+$ cells, $n=6$ ). On P21, MBP immunostaining was significantly reduced in LPS-treated rat brains, which was associated with a reduced number of APC-CC1 + mature OLs (Fig. 2). LPS treatment also resulted in an increased number of pyknotic cells in the cortex at P6 (primarily in layers II and III) and P8 (primarily in the cingular cortex), as shown by their condensed and fragmented nuclei in Nissl-stained sections (Fig. $3 B$ and $E$ ). Cell death in the cortex was further
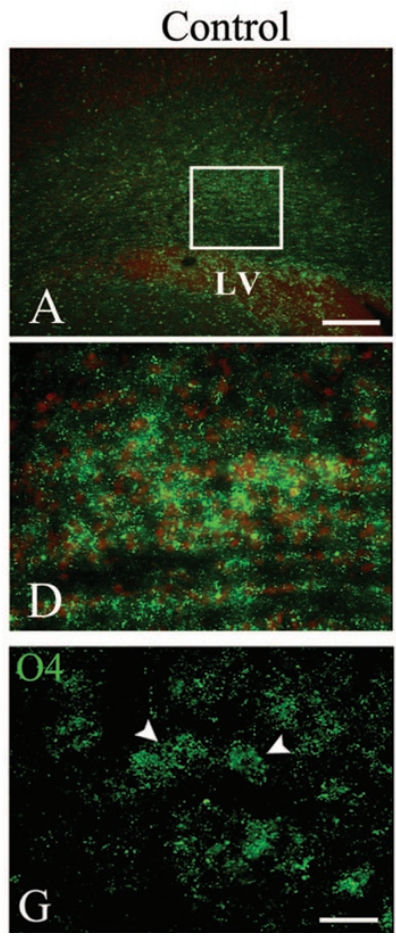
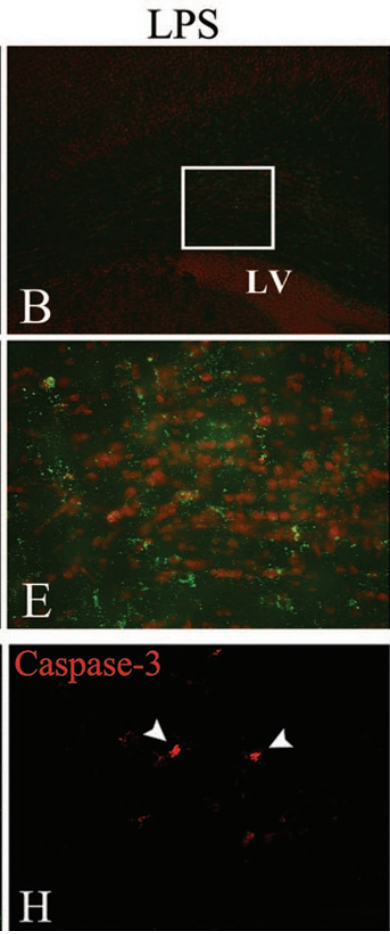
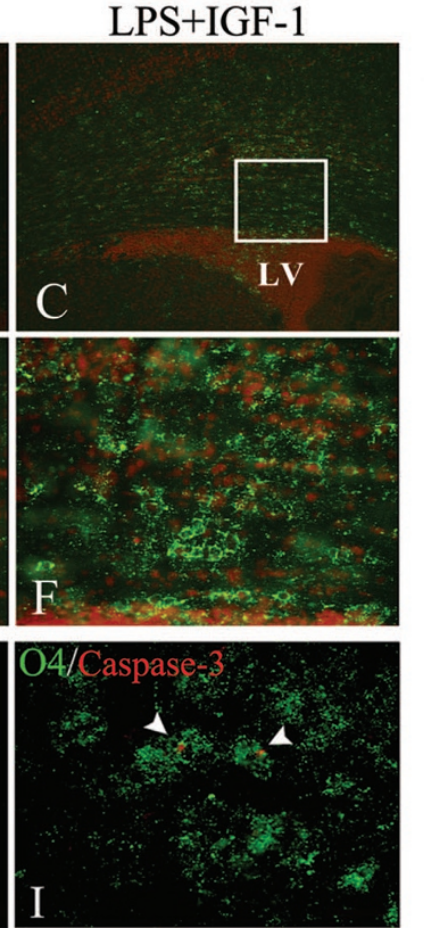

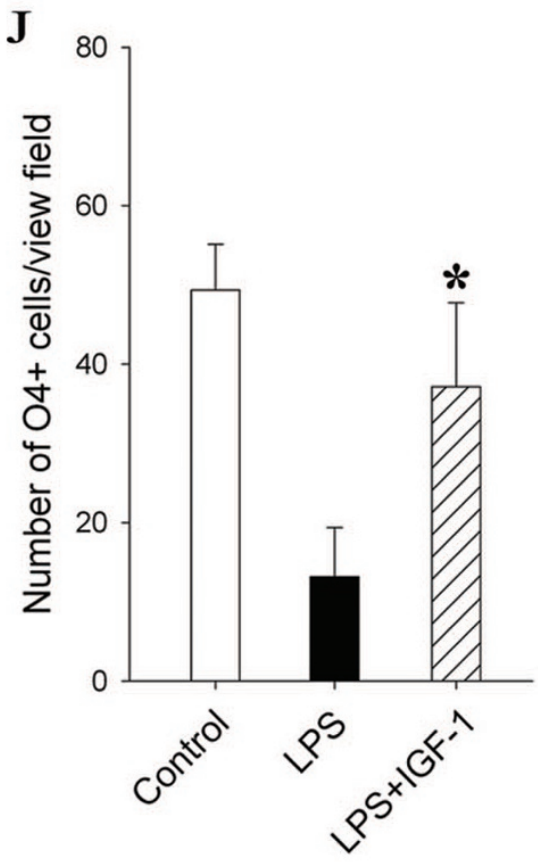

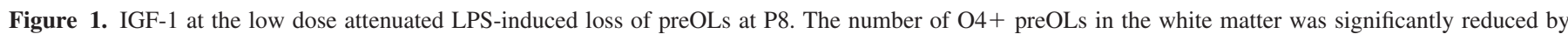

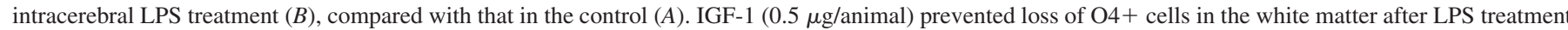

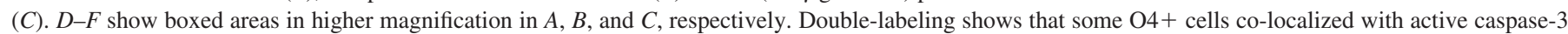

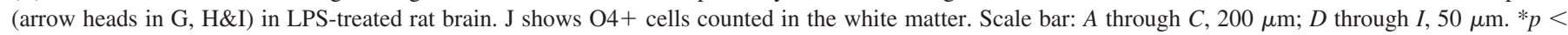
0.01 vs LPS treatment. 

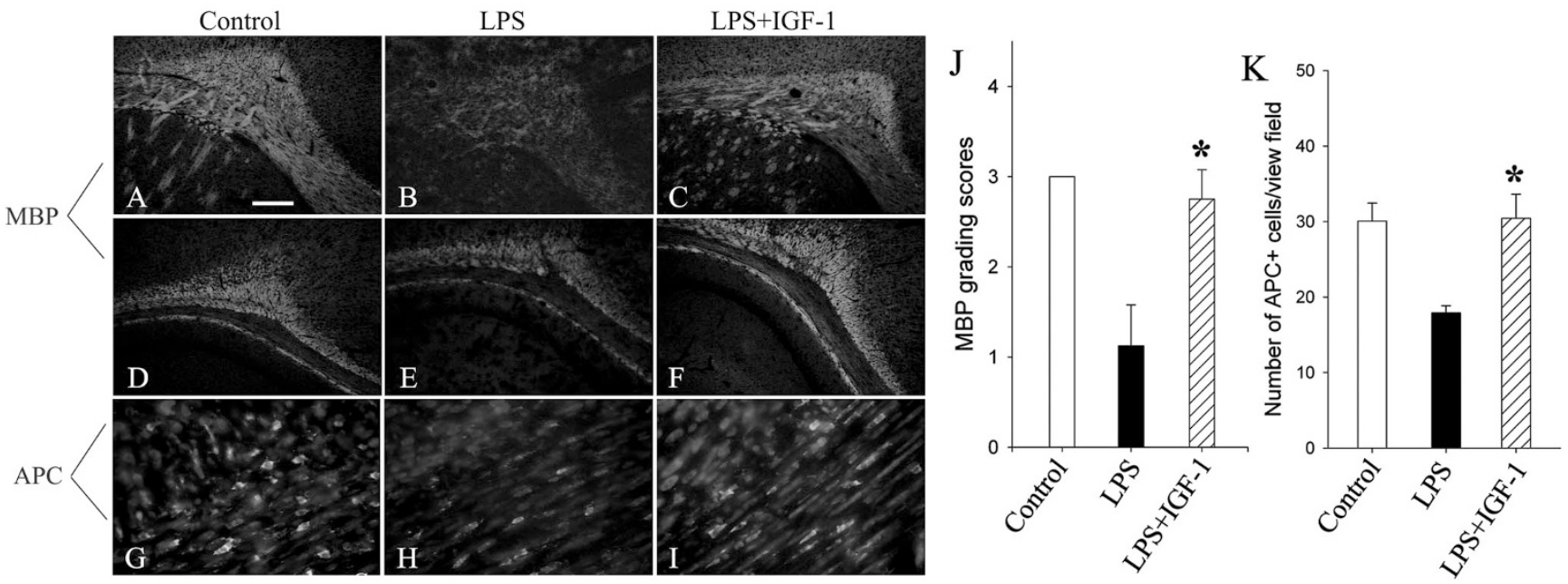

Figure 2. IGF-1 at the low dose attenuated LPS-induced hypomyelination and decreased the number of mature OLs. The extent of myelination was estimated by MBP immunohistochemistry at both the bregma $(A-C)$ and the dorsal hippocampal level $(D, E$ and $F)$. The mature OLs were identified using APC antibody $(G-I)$. MBP expression was quantified using a grading method with scores from 0 to 3 , where 0 , no staining; 1 , weak but detectable staining; 2 , reduced staining compared with 3 but stronger than 1; and 3, normal. LPS treatment resulted in a significant decrease of MBP scores in the white matter $(J)$. The number of APC + mature OLs was also reduced in LPS treatment compared with that in the control $(K)$. IGF-1 significantly attenuated LPS-induced reduction in MBP expression as well as decrease of mature OLs number. Scale bar: $A$ through $F, 200 \mu \mathrm{m} ; G$ through $I, 50 \mu \mathrm{m} . * p<0.01 v s$ LPS treatment.
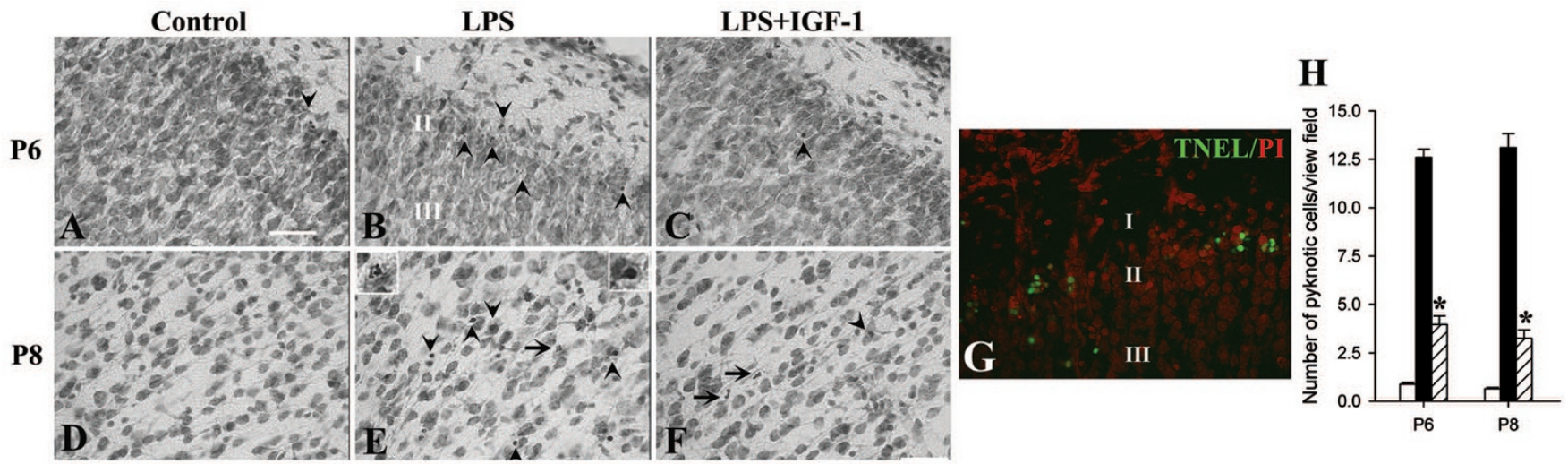

Figure 3. IGF-1 at the low dose protected against LPS-induced cell death in the gray matter. LPS treatment resulted in an increased number of pyknotic cells in the cortex at P6 ( $B$, layer II and III) and P8 (E, cingular subcortex), compared with the controls ( $A$ and $D$ ). IGF-1 significantly decreased the number of pyknotic cells at both P6 and P8 ( $C$ and $F$ ). Pyknotic cells (arrow heads) are distinguished from PMNs (arrows) by their condensed/fragmented nuclei as shown in the inserts in $E$ (PMN, up left; pyknotic cell, up right). The pyknotic cells shown in $B$ were also positive for TUNEL ( $G$ ). Pyknotic cell counting is shown in $\mathrm{H}$ (open bars: control; black bars: LPS; striped bars: LPS +IGF-1). Scale bar: $50 \mu \mathrm{m}$. $* p<0.01$ vs LPS treatment.

identified using TUNEL (Fig. 3G). IGF-1 at a lower dose $(0.5$ $\mu \mathrm{g} /$ animal) significantly prevented LPS-induced loss of preOLs (Fig. $1 C$ and $F$ ), reduction of MBP immunostaining (Fig. $2 C$ and $F)$ and increase in the number of pyknotic cells in the cortex (Fig. $3 C$ and $F$ ) when compared with LPS treatment alone.

IGF-1 at the low dose had no effect on LPS-activated microglia and astrocytes, but significantly enhanced LPSinduced polymorphonuclear leukocyte (PMN) recruitment to the brain parenchyma.

Consistent with our previous reports (4), the number of $\mathrm{ED}+$ activated microglia/macrophage and GFAP+ astrocytes significantly increased in the LPS-treated rat brain compared with the control. IGF-1 had no effect on either LPS-induced microglial activation (Fig. $4 A-C$, ED1 immunostaining) or astrogliosis (Fig. $4 D-F$, GFAP immunostaining), and did not change IL- $1 \beta$ expression $(430.3 \pm 37.5 \mathrm{pg} / \mathrm{mg}$ total protein for LPS, $511.9 \pm 38.6 \mathrm{pg} / \mathrm{mg}$ total protein for LPS + IGF-1, $n=8, p>0.05$ ).

Despite no effects on glial cells, IGF-1 significantly enhanced LPS-induced PMN recruitment to the brain parenchyma (Fig. 4G-I, CD43 immunostaining). At P6, more PMNs were found in LPS + IGF-1-treated brain white matter than LPS treatment alone; at P8, the number of PMNs remained higher in the LPS-treated brain, while it quickly declined in the LPS + IGF-1 treated brain (Fig. $4 J$ ). No PMNs were found in the brains of the control or the IGF-1 only treatment group.

Coapplication of LPS with IGF-1 at the low dose resulted in brain hemorrhage. The more serious adverse effect of coapplication of LPS and IGF-1 at the low dose was a significant increase of intracerebral hemorrhage (Fig. 5, upper panel). At P6, only one of eight (12.5\%) rat brains treated with LPS showed two small hemorrhages around the white matter 

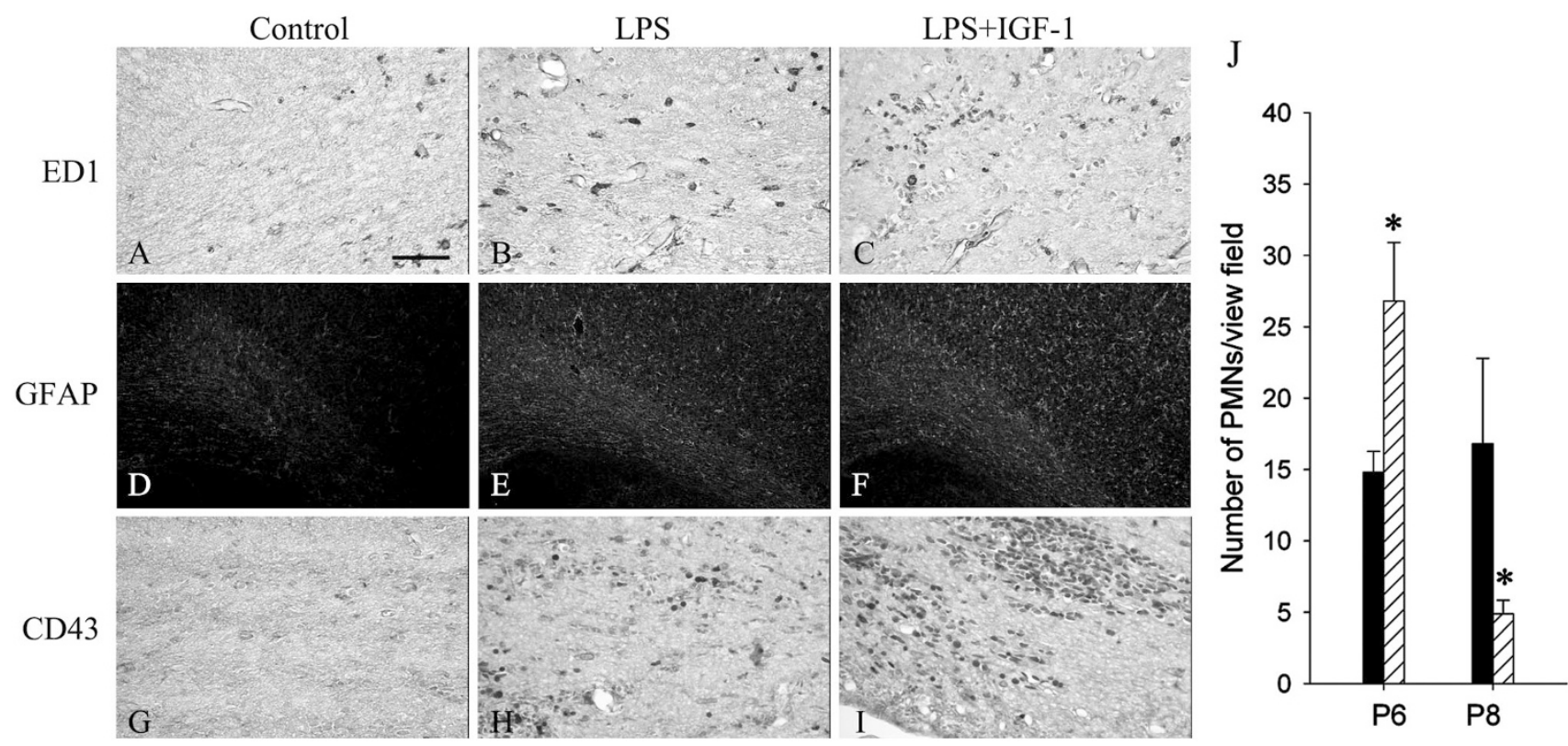

Figure 4. IGF-1 at the lower dose had no effect on LPS-induced microglia and astrocyte activation, but significantly enhanced PMN infiltration into brain parenchyma. LPS treatment resulted in activation of microglia/macrophages ( $B$, ED1 immunostaining) and astrocytes ( $E$, GFAP immunostaining), and infiltration of PMNs ( $H$, CD43 immunostaining) in the white matter, compared with that of the control $(A, D$, and $G$, respectively) at P6. IGF-1 had no effect on LPS-induced microglia/macrophage activation $(C)$ and astrogliosis $(F)$, but significantly potentiated LPS-induced PMN infiltration $(I)$. On P8, the number of PMNs in LPS + IGF-1 group (striped bar) quickly declined to a level lower than LPS treatment alone (black bar) $(J)$. No PMNs were found in any of the rat brain treated with IGF-1 alone (not shown). Scale bar: $A-C$ and $G-I, 50 \mu \mathrm{m} ; D-F, 200 \mu \mathrm{m} . * p<0.05 v s$ LPS treatment.

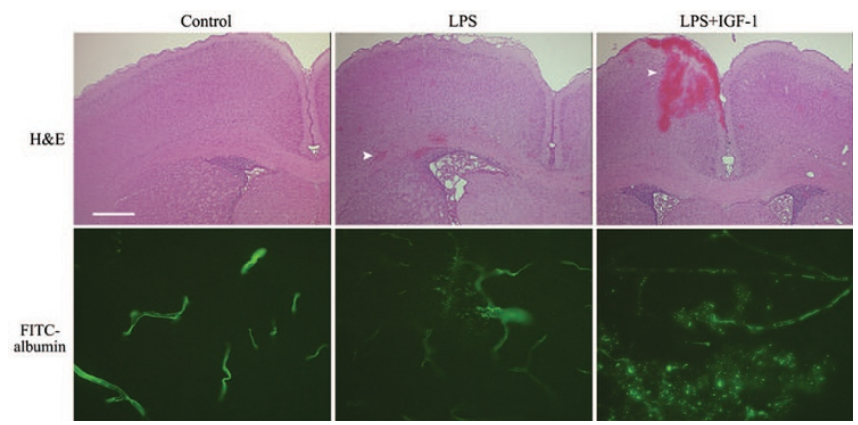

Figure 5. IGF-1 at the low dose potentiated LPS-induced brain hemorrhage and BBB disruption. Representative HE staining at the bregma level shows mild hemorrhage (indicated by an arrow head) in one of the LPS-treated rat brains at P6. LPS + IGF-1 ( $0.5 \mu \mathrm{g} / \mathrm{animal})$ resulted in worse brain hemorrhage, as shown by a large area of hemorrhage in the cortex (hemorrhage in other brain areas was not shown). FITC-albumin was found to be restricted to the blood vessels in the control rat brain, but to be diffused into brain parenchyma from some blood vessels in the periventricular white matter in LPS-treated rat brain. IGF-1 further potentiated LPS-induced leakage of FITC-albumin. Scale bar in H\&E sections: $500 \mu \mathrm{m}$; in FITC-albumin sections: $50 \mu \mathrm{m}$.

in HE-stained sections. Treatment with LPS + IGF-1 markedly increased the incidence (100\%, eight of eight rat brains) and severity of brain hemorrhage. The size of hemorrhage ranged from small to very large and was found in every brain structure such as cortex, ventricles, white matter, subarachnoid space, and basal ganglia around the third ventricle (only a large area of hemorrhage in the cortex was shown in a H\&E stained section in Fig. 5). At P8, none of the LPS-treated rats showed any signs of hemorrhage, but it was still present in some of the LPS + IGF-1 treated rat brains.

IGF-1 at the low dose enhanced LPS-induced breakdown of BBB. To find out if PMN infiltration is associated with changes of $\mathrm{BBB}$ integrity, we investigated the permeability of the BBB using FITC-labeled albumin. As shown in the lower panel of Fig. 5, the green fluorescence was only visible in blood vessels but not in the brain parenchyma in the control rate brain. The leakage of FITC-labeled albumin from blood vessels to the parenchyma was seen in LPS-treated rat brains that was primarily located in the periventricular white matter. Coapplication of IGF-1 with LPS further increased BBB permeability as indicated by the increase in intensity of the fluorescence in the brain parenchyma. No change of BBB integrity was found in the rat brain treated with IGF-1 alone compared with the controls (data not shown).

Treatment with LPS and higher doses of IGF-1 resulted in higher mortality in the animals, and massive blood cell infiltration and severe hemorrhage in the rat brains.

At higher doses (5 and $25 \mu \mathrm{g} / \mathrm{animal}$ ), coapplication of IGF-1 with LPS resulted in a high mortality. The mortality is similar between $5 \mu \mathrm{g}(53.3 \%, 16$ of 30 animals) and $25 \mu \mathrm{g}$ (51.6\%, 16 of 31 animals). No animals died from treatment with LPS or IGF-1 alone. The surviving rats treated with LPS and higher doses of IGF-1 showed severe brain damage, which was associated with increased intracerebral hemorrhage (Fig. 6A, a blood clot was shown in 3rd dorsal ventricle) and leukocyte infiltration. In some rat brains, the blood cells seemed to originate from the ventral 3 rd ventricle, infiltrating into the surrounding tissue and causing damage to the septal region and thalamus/hypothalamus (Fig. $6 B$ and $C$ ). On P8, the lateral ventricles of rats treated with LPS plus higher doses of IGF-1 were dramatically dilated and associated with damage of the periventricular white matter (Fig. 6I), as shown in Nissl staining. The numbers of both pyknotic cells and PMNs were remarkably increased in the white matter of LPS + 


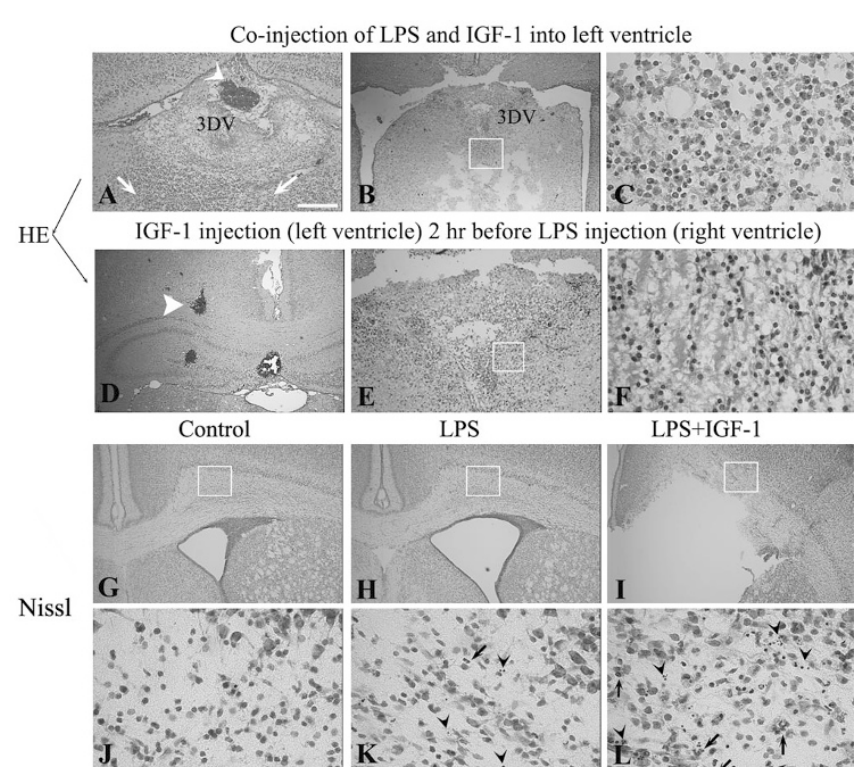

Figure 6. IGF-1 at a higher dose ( $5 \mu \mathrm{g} / \mathrm{animal})$ enhanced LPS-induced brain injury. Co-injection of LPS with the higher dose of IGF-1 resulted in severe hemorrhage inside the third dorsal ventricle (3DV) ( $A$, arrow head), and massive leukocyte infiltration ( $A$, arrows) surrounding the $3 \mathrm{DV}$. In some animals, the brain tissue around the 3DV (especially the ventral part) was severely damaged and totally replaced with infiltrated inflammatory cells ( $B$, boxed area is highlighted in $C$ ). A separate injection of LPS with IGF-1 (injected $2 \mathrm{~h}$ before LPS) resulted in similar pathology (brain hemorrhage shown in $D$, tissue loss shown in $E$ and leukocyte infiltration shown in $F$ ). At P8, the Nissl-stained sections at the bregma level show enlarged lateral ventricle $(H)$ and cell death (arrow heads in $K$ ) in LPS-treated rat brain, compared with the control ( $G$ and $J$ ). IGF-1 deteriorates LPS-induced ventricle dilation $(I)$. A marked increase in both pyknotic cells (arrow heads) and PMNs (arrows) was found in the LPS + IGF-1 treated rat brain white matter (L). Scale bar: in $A, B, D, E, G, H$, and $I, 200 \mu \mathrm{m} ; C, F, J-L: 50 \mu \mathrm{m}$.

IGF-1-treated rat brain (Fig. 6L), compared with that with LPS treatment alone (Fig. $6 K$ ).

Because higher doses of IGF-1 are known to decrease blood glucose level, the rat blood glucose level was measured using a glucose meter (Bayer Healthcare LLC, Mishawaka, IN) $24 \mathrm{~h}$ after treatment. We did not find any change of blood glucose levels among all treatment groups (control $=103.8 \pm 1.3$ $\mathrm{mg} / \mathrm{dL}$, LPS $=99.4 \pm 5.5 \mathrm{mg} / \mathrm{dL}$, IGF- $1=107.5 \pm 4.9$ $\mathrm{mg} / \mathrm{dL}$, and LPS $+\mathrm{IGF} 1=91.0 \pm 9.2 \mathrm{mg} / \mathrm{dL}$. Mean $\pm \mathrm{SEM}$, $n=6)$.

We also tried to use different treatment regimens. In one set of experiments, IGF-1 $(5 \mu \mathrm{g} / \mathrm{mL})$ was first injected into the left ventricle and $2 \mathrm{~h}$ later, LPS was injected into the right ventricle. The mortality ( $44.4 \%$, eight of 18 rats), brain hemorrhage, leukocyte infiltration, and brain damage (Fig. 6D-F) of the separate injection regimen were all similar to those found in the co-injection.

\section{DISCUSSION}

This study demonstrated that IGF-1 at a low dose significantly prevented LPS-induced preOL loss (Fig. 1) and MBP reduction (Fig. 2) in the white matter, as well as cell death in the cortex (Fig. 3). Surprisingly, IGF-1 at higher doses increased the detrimental effects of LPS. This deterioration seems to be through an interaction of IGF-1 with LPS- mediated brain inflammation, since no such effects were observed in rat brain treated with IGF-1 alone. To our knowledge, this is the first report showing that IGF-1 enhances LPS-induced BBB breakdown, leukocyte infiltration, intracerebral hemorrhage, and brain damage.

Although there are significant numbers of in vitro studies demonstrating that IGF-1 is able to protect OLs from various insults (6-8), fewer studies have been performed in animal models of PVL. In a neonatal hypoxia-ischemia model, IGF-1 has been shown to protect against neuronal/OL damage due to hypoxia-ischemia $(9-11)$. Our current study is the first to test if IGF-1 provides neuroprotection against LPS-induced brain damage. LPS induced degeneration of $\mathrm{O} 4+$ preOLs in white matter, as shown by the co-localization of $\mathrm{O} 4$ with active caspase-3 antibodies (Fig. 1), and subsequently, LPS reduced the number of mature OLs and caused hypomyelination (Fig. 2). In addition, we also observed cell death in the cortex (Fig. 3 ), supporting the recent recognition that PVL is also associated with neuronal damage (2). The protection of both white and gray matter was clearly demonstrated when IGF-1 was used at a low dose; this could be attributed to its potent survival effect on both neurons and OLs. However, some adverse effects such as an increase in PMN recruitment and BBB permeability also occurred at the low dose, and these effects became much more pronounced at higher doses. The brain damage observed with LPS + IGF-1 at higher doses was clearly associated with an overwhelming leukocyte infiltration into the brain parenchyma (Fig. 6).

An important finding of this study is that in the presence of LPS, the beneficial or detrimental effects of IGF-1 depend on its dose. The dose effects of IGF-1 in neuroprotection have been reported in several studies. Brywe et al. (9) and Wood et al. (10) reported that IGF-1 protects against hypoxia-ischemic brain damage only at a high dose $(50 \mu \mathrm{g} / \mathrm{animal})$. Though intranasally delivered, Vig et al. (15) demonstrated that IGF-1 at a total dose of $600 \mu \mathrm{g}$ over a 48 -h period protects against hypoxic-ischemic brain damage. Contrarily, Cao et al. (16) showed that IGF-1 at a lower dose ( $3 \mu \mathrm{g}$ per animal), but not a high dose (30 $\mu \mathrm{g}$ per animal), significantly prevented loss of OLs and myelin in fetal sheep subjected to hypoxia ischemia. Interestingly, there are also a few studies showing that IGF-1 failed to prevent and even exacerbated neuronal damage. For example, targeted expression of IGF-1 by adenovirus failed to protect OLs from experimental autoimmune encephalomyelitis in the spinal cord (17). More recently, Escartin et al. (18) reported that IGF-1 failed to alleviate motor impairment and weight loss in a rat model of Huntington's disease. At a high dose, IGF-1 exacerbated neuronal damage induced by 3-nitropropionic acid. These studies, together with our current data, suggest that the dose effects of IGF-1 in neuroprotection may also depend on specific animal models and pathologic conditions. At higher doses, IGF-1 may exacerbate neuronal damage in some cases.

The mechanism(s) of how IGF-1 recruits more PMNs into the brain is not clear but is possibly due to increased permeability of BBB and/or up-regulation of certain chemotactic and adhesion molecules. Indeed, it has been shown that IGF-1 increases the intercellular adhesion molecule- 1 gene in endo- 
thelial cells through activation of NFkB (19), and furthermore, potentiates TNF $\alpha$-induced activation of c-Jun/NFkB as well as up-regulation of adhesion molecule expression (20). Similarly, IGF-1 has been shown to induce (21), or enhance TNF $\alpha$-mediated expression of IL-8 (22), a potent chemoattractant for PMNs. These studies suggest that IGF-1 may interact with LPS-induced proinflamatory cytokines to enhance chemokine production and in turn, increase PMN infiltration into brain parenchyma. Our observation that the brain inflammation/damage is similar with co-injection or separate injection of LPS and IGF-1 indicates that the adverse effects were not due to a physical interaction of LPS and IGF-1, but rather an interaction between LPS-induced inflammatory mediators and IGF-1. The mechanism of increased BBB permeability by IGF-1 is not clear, but there is a study suggesting a potential link between IGF-1 and BBB dysfunction, in which IGF-1 regulates zona occludens-1 protein (23), one of the proteins found in tight junctions of the BBB. Because the BBB is not well developed in neonatal rats (24), it is possible that the adverse effect of IGF-1 is specific for the developing brain, but this needs to be confirmed in a future study.

IGF-1 has been used in many clinical trials. It seems there are no serious side effects in using human recombinant IGF-1. The most common side effect of IGF-1 is hypoglycemia when it is used at a high dose (25). This is not the case in our study as the blood glucose level was not different between LPS and LPS + IGF-1-treated animals. The detrimental effects of IGF-1 are apparently not related to IGF-1 by itself and thus should not be considered as a side effect. However, this study does provide alarming evidence that in an acute inflammatory condition, IGF-1 may have severe, harmful effects on the developing brain.

\section{REFERENCES}

1. Volpe JJ 2003 Cerebral white matter injury of the premature infant-more common than you think. Pediatrics 112:176-180

2. Khwaja O, Volpe JJ 2008 Pathogenesis of cerebral white matter injury of prematurity. Arch Dis Child Fetal Neonatal Ed 93:F153-F161

3. Leviton A, Dammann O 2004 Coagulation, inflammation, and the risk of neonatal white matter damage. Pediatr Res 55:541-545

4. Pang Y, Cai Z, Rhodes PG 2003 Disturbance of oligodendrocyte development, hypomyelination and white matter injury in the neonatal rat brain after intracerebral injection of lipopolysaccharide. Brain Res Dev Brain Res 140:205-214

5. McQuillen PS, Ferriero DM 2004 Selective vulnerability in the developing central nervous system. Pediatr Neurol 30:227-235
6. Pang Y, Zheng B, Fan LW, Rhodes PG, Cai Z 2007 IGF-1 protects oligodendrocyte progenitors against TNFalpha-induced damage by activation of PI3K/Akt and interruption of the mitochondrial apoptotic pathway. Glia 55:1099-1107

7. Cui QL, Zheng WH, Quirion R, Almazan G 2005 Inhibition of Src-like kinases reveals Akt-dependent and -independent pathways in insulin-like growth factor I-mediated oligodendrocyte progenitor survival. J Biol Chem 280:8918-8928

8. Ness JK, Scaduto RC Jr, Wood TL 2004 IGF-I prevents glutamate-mediated bax translocation and cytochrome $\mathrm{C}$ release in $\mathrm{O} 4+$ oligodendrocyte progenitors. Glia 46:183-194

9. Brywe KG, Mallard C, Gustavsson M, Hedtjärn M, Leverin AL, Wang X, Blomgren K, Isgaard J, Hagberg H 2005 IGF-I neuroprotection in the immature brain after hypoxiaischemia, involvement of Akt and GSK3beta? Eur J Neurosci 21:1489-1502

10. Wood TL, Loladze V, Altieri S, Gangoli N, Levison SW, Brywe KG, Mallard C, Hagberg H 2007 Delayed IGF-1 administration rescues oligodendrocyte progenitors from glutamate-induced cell death and hypoxic-ischemic brain damage. Dev Neurosci 29:302-310

11. Zhong J, Zhao L, Du Y, Wei G, Yao WG, Lee WH 2009 Delayed IGF-1 treatment reduced long-term hypoxia-ischemia-induced brain damage and improved behavior recovery of immature rats. Neurol Res 31:483-489

12. Johnston MV, Hagberg H 2007 Sex and the pathogenesis of cerebral palsy. Dev Med Child Neurol 49:74-78

13. Lin S, Fan LW, Pang Y, Rhodes PG, Mitchell HJ, Cai Z 2005 IGF-1 protects oligodendrocyte progenitor cells and improves neurological functions following cerebral hypoxia-ischemia in the neonatal rat. Brain Res 1063:15-26

14. Carvey PM, Zhao CH, Hendey B, Lum H, Trachtenberg J, Desai BS, Snyder J, Zhu YG, Ling ZD 2005 6-Hydroxydopamine-induced alterations in blood-brain barrier permeability. Eur J Neurosci 22:1158-1168

15. Vig PJ, Subramony SH, D'Souza DR, Wei J, Lopez ME 2006 Intranasal administration of IGF-1 improves behavior and Purkinje cell pathology in SCA1 mice. Brain Res Bull 69:573-579

16. Cao Y, Gunn AJ, Bennet L, Wu D, George S, Gluckman PD, Shao XM, Guan J 2003 Insulin-like growth factor (IGF)-1 suppresses oligodendrocyte caspase-3 activation and increases glial proliferation after ischemia in near-term fetal sheep. J Cereb Blood Flow Metab 23:739-747

17. Genoud S, Maricic I, Kumar V, Gage FH 2005 Targeted expression of IGF-1 in the central nervous system fails to protect mice from experimental autoimmune encephalomyelitis. J Neuroimmunol 168:40-45

18. Escartin C, Boyer F, Bemelmans AP, Hantraye P, Brouillet E 2007 IGF-1 exacerbates the neurotoxicity of the mitochondrial inhibitor 3NP in rats. Neurosci Lett 425:167-172

19. Balaram SK, Agrawal DK, Edwards JD 1999 Insulin like growth factor-1 activates nuclear factor-kappaB and increases transcription of the intercellular adhesion molecule-1 gene in endothelial cells. Cardiovasc Surg 7:91-97

20. Che W, Lerner-Marmarosh N, Huang Q, Osawa M, Ohta S, Yoshizumi M, Glassman M, Lee JD, Yan C, Berk BC, Abe J 2002 Insulin-like growth factor-1 enhances inflammatory responses in endothelial cells: role of Gab1 and MEKK3 in TNFalpha-induced c-Jun and NF-kappaB activation and adhesion molecule expression. Circ Res 90:1222-1230

21. Kooijman R, Coppens A, Hooghe-Peters E 2003 IGF-I stimulates IL-8 production in the promyelocytic cell line HL-60 through activation of extracellular signalregulated protein kinase. Cell Signal 15:1091-1098

22. Vallée S, Fouchier F, Brémond P, Briand C, Marvaldi J, Champion S 2003 Insulin-like growth factor-1 downregulates nuclear factor kappa B activation and upregulates interleukin- 8 gene expression induced by tumor necrosis factor alpha. Biochem Biophys Res Commun 305:831-839

23. Mauro L, Bartucci M, Morelli C, Andò S, Surmacz E 2001 IGF-I receptor-induced cell-cell adhesion of MCF-7 breast cancer cells requires the expression of junction protein ZO-1. J Biol Chem 276:39892-39897

24. Tonra JR, Mendell LM 1997 Rabbit IgG distribution in skin, spinal cord and DRG following systemic injection in rat. J Neuroimmunol 80:97-105

25. Kovacs GT, Worgall S, Schwalbach P, Steichele T, Mehls O, Rosivall L 1999 Hypoglycemic effects of insulin-like growth factor-1 in experimental uremia: can concomitant growth hormone administration prevent this effect? Horm Res 51:193-200 